\title{
Special issue on visual information retrieval
}

\author{
Michael S. Lew ${ }^{1}$
}

Published online: 27 January 2016

(C) Springer-Verlag London 2016

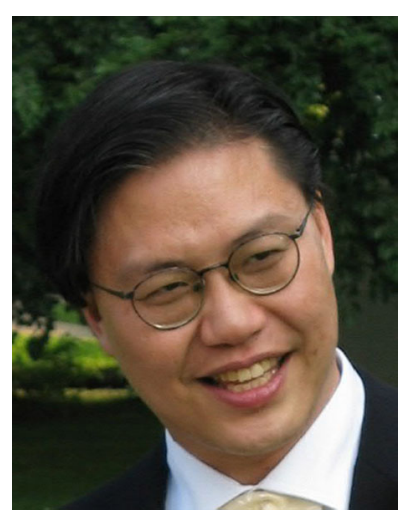

This special issue provides an overview of important work and the leading research directions in the area of visual information retrieval. With the flood of images and video from diverse sources (e.g., smartphones, NetFlix, FLICKR, Amazon, Instagram, Twitter, etc.), there is a great need to be able to browse, index and search through the mountain of visual data. The papers within this special issue are amongst the top scientific work worldwide in visual information retrieval and provide a snapshot of the state-of-the-art.

All submissions were triple peer-reviewed and then given a summary review by a member of the editorial board. This year each paper had to be recommended by a member of the IJMIR editorial board and a program chair from ICMR. I would like to thank Tat-Seng Chua, Yu-Gang Jiang, Cees Snoek and Nunu Vansconcelos for their assistance in the paper selection process.

One of the current frontier areas is searching for video over the Internet. In the paper, "Text-to-video: a semantic search

\author{
Michael S. Lew \\ mlew@liacs.nl \\ 1 Leiden University, Leiden, The Netherlands
}

engine for internet videos" by Lu Jiang, Shoou-I Yu, Deyu Meng, Teruko Mitamura and Alexander G. Hauptmann, the authors present a semantic video search system which does not use any user meta-data and also had the best performance from the well known NIST TRECVID 2014 competition. They also provide several interesting recommendations for future video search systems.

Another leading approach to video search is to make the search adaptive to the user, that is, to use any multi-modal data linked to the user. In the paper, "User-adaptive image retrieval via fusing pointwise and pairwise labels" by Lin Chen, Peng Zhang and Baoxin $\mathrm{Li}$, the authors propose a hybrid ranking framework which results in significant performance improvements. They also found that their online learning approach has superior performance as compared to batch learning.

In the classic visual learning method there needs to be substantial and time consuming manual annotation. One of the current trends seeks to automatically discover relevant image groups without relying on manual annotation. In the paper, "Bundling centre for landmark image discovery" by Qian Zhang and Guoping Qiu, the authors present several ideas toward automatic discovery of landmark images which are shown in their experiments to be robust to outliers and have state-of-the-art precision.

Social computing is one of the important new paradigms and provides new interaction possibilities, types of data and challenges. In the paper, "MGraph: multimodal event summarization in social media using topic models and graphbased ranking" by Manos Schinas, Symeon Papadopoulos, Yiannis Kompatsiaris and Pericles A. Mitkas, the authors describe a novel summarization framework for social media. For a set of social media posts, it attempts to automatically find a subset which maximizes the relevance and minimizes redundancy. The experiments reveal that their method outper- 
forms the competitive approaches regarding summarization precision.

The field of visual information retrieval has been rapidly evolving. The last decade has seen enormous growth in the accuracy, scale and scope of image and video retrieval techniques, from detecting a dozen visual concepts in databases containing twenty thousand images in 2005 to the current bar of understanding thousands of visual concepts and indexing databases holding hundreds of millions of images (and video). It will be exciting to see what the next decade has in store of us. 